\title{
Digit ratio (2D:4D) in newborns: Influences of prenatal testosterone and maternal environment
}

\author{
T. Ventura ${ }^{\text {a,* }}$, M.C. Gomes $^{\text {b }}$, A. Pita ${ }^{\text {a }}$, M.T. Neto ${ }^{\text {a,d }}$, A. Taylor ${ }^{\mathrm{c}}$ \\ a Hospital Dona Estefânia, Centro Hospitalar de Lisboa Central EPE, Rua Jacinta Marto, 1169-045 Lisbon, Portugal \\ ${ }^{\mathrm{b}}$ Faculty of Sciences, University of Lisbon, Campo Grande, 1749-016 Lisbon, Portugal \\ c School of Biomedical Sciences, King's College London, Franklin Wilkins Building, Waterloo, London SE1 9NH, United Kingdom \\ d Faculty of Medical Sciences, Universidade Nova de Lisboa, Campo dos Mártires da Pátria Lisboa, Portugal
}

\section{A R T I C L E I N F O}

\section{Article history:}

Received 8 June 2012

Received in revised form 26 August 2012

Accepted 28 August 2012

\section{Keywords:}

Newborn

Testosterone

2D:4D

Digit ratio

Sexual dimorphism

\begin{abstract}
A B S T R A C T
Introduction: The 2D:4D digit ratio is sexually-dimorphic, probably due to testosterone action through the perinatal period. We characterize the 2D:4D ratio in newborn (NB) infants, in between the pre- and postnatal surges of testosterone, and relate it to the mother's 2D:4D and to testosterone levels in the amniotic fluid (AF). Subjects and methods: Testosterone was assayed in samples of maternal plasma and AF collected at amniocentesis. Shortly after birth, 106 NBs and their mothers were measured for 2D:4D ratio.

Results: NB males had lower mean 2D:4D ratios than females but this dimorphism was significant only for the left hand (males: 0.927 ; females: $0.950 ; \mathrm{p}=0.004$ ). Mothers who had sons had lower 2D:4D ratios than those who had daughters and the mother's 2D:4D were higher than those of NBs regardless of sex. Both hands of NB females were negatively correlated with AF testosterone and positively correlated with the mother's $2 \mathrm{D}: 4 \mathrm{D}$, but males showed no significant associations. Maternal plasma testosterone also showed a negative weak correlation with NB's digit ratio in both sexes.

Conclusions: Sexual dimorphism at birth was only significant for the left hand, in contrast with reports of greater right hand dimorphism, suggesting that postnatal testosterone is determinant for 2D:4D stabilization. The lower 2D:4D ratios in mothers who had sons support claims that hormone levels in parents are influential for determining their children's sex. NB female's digit ratio, but not males', was associated to the level of AF testosterone. The mother's 2D:4D ratios were positively correlated with their daughters' 2D:4D, but the same was not observed for male NBs, suggesting that prenatal testosterone levels in male fetus lead their 2D:4D ratios to stray from their mothers' with high individual variability.
\end{abstract}

(c) 2012 Elsevier Ireland Ltd. All rights reserved.

\section{Introduction}

In humans, the ratio of the index finger (2D) to the ring finger (4D) is sexually dimorphic, as women have a larger mean 2D:4D ratio than men [1,2], a tendency previously described in several populations [3]. As many somatic sex differences result from prenatal androgen masculinization, it has been suggested that prenatal testosterone is responsible for the sex difference in mean 2D:4D ratio, with the latter being negatively correlated with testosterone [2,4]. Strong evidence for the role of androgen comes from the association between the 2D:4D ratio and congenital adrenal hyperplasia (CAH), a condition of elevated androgen production, as both males and females with CAH have smaller 2D:4D than controls [5,6]. Also female dizygotic twin fetuses growing next to males were found to have lower ratio than the ones growing next to females [7,8] suggesting the influence of some level of androgen diffusion. This has further

\footnotetext{
* Corresponding author at: Centro Hospitalar de Lisboa Central EPE, Rua Jacinta Marto, 1169-045 Lisbon, Portugal. Tel.: + 351 914741166(TM).

E-mail address: tventura@sapo.pt (T. Ventura).
}

been corroborated by work showing that variation in 2D:4D correlates with androgen receptor activity in men, as indicated by the number of CAG repeats in the androgen receptor allele [9]. More recently, an experimental study [10] with a mouse strain known to be sexually dimorphic, provided compelling evidence that prenatal testosterone and estrogen determine mouse 2D:4D during embryonic development, with sex steroids controlling digit development by regulating the skeletogenic expression of at least 19 genes. The 2D:4D ratio at birth should thus reflect a genetic background subjected to a given level of prenatal androgen and estrogen exposure.

Garn et al. [11] measured human embryos and fetuses to find that phalangeal length ratios later found in adulthood are attained early in gestation, by the end of the 13th week. Since then some authors assumed that the 2D:4D sexual dimorphism is established during early prenatal development, with the $2 \mathrm{D}: 4 \mathrm{D}$ ratio remaining stable thereafter $[2,3,5,11,12]$. But if sex steroids determine digit growth, their effect may not be limited to the prenatal period, as exposure to testosterone proceeds non-uniformly across the first years of life. Human perinatal testosterone production comprises three peaks, a mid-gestational and two postnatal peaks [13]. The mid-gestational 
peak takes place between weeks 14 and 18, although most prenatal androgen effects occur between weeks 7 and 12 [13]. Serum testosterone rises to a first postnatal peak around two months of age, falling again to childhood levels by 4 to 6 months. A third (second postnatal) peak is found from puberty onwards to adulthood. Thus secondary sex differences arising prior to puberty, namely the 2D:4D ratio, are usually attributable to the effects of prenatal and/or early postnatal testosterone production.

Two longitudinal studies $[14,15]$ provided solid evidence that the digit ratios increase from infancy to adulthood, especially between ages one and five, although the magnitude of the change is small. Similarly, Galis et al. [16] working with 327 deceased human fetuses (14 to 42 weeks old) reported that the prenatal 2D:4D in both sexes was lower than has been reported for children and adults, suggesting that it may increase after birth. More recently, McIntyre and Alexander [17], in a sample of 74 young children (3-5 months old) also found relatively low 2D:4D values, and Knickmeyer et al. [18] followed $2 \mathrm{D}: 4 \mathrm{D}$ during the postnatal testosterone surge $(0-2$ years old) to conclude that 2D:4D ratios change, apparently modulated by postnatal testosterone, with such individual variability that led the authors to suggest that 2D:4D measured in early childhood may not correlate well with prenatal exposure to testosterone.

We are not aware of reports of 2D:4D ratios focused upon the first days in the life of newborn (NB) infants, a narrow time window in between the pre- and postnatal peaks of testosterone production, when only effects of prenatal genetic and hormonal interaction should be apparent. The purpose of this study is to examine dimorphism in the 2D:4D digit ratio at delivery (first $48 \mathrm{~h}$ ) and to report on how the digit ratio of the NB correlates with mother's 2D:4D ratios and with testosterone in the amniotic fluid (AF) and in maternal plasma, both evaluated at the time of amniocentesis (16-23 weeks of pregnancy).

\section{Methods}

Mothers were referred to the hospital for karyotyping and were recruited at the time of amniocenteses, as part of a prospective study conducted at the maternity of Dona Estefânia Hospital (HDE) in Lisbon, between December 2009 and June 2011. The study was approved by the Hospital Ethics Committee and written informed consents were obtained for all the procedures, as approved by the Committee. Mothers willing to deliver at HDE maternity were invited to participate. Only healthy mothers with singleton spontaneous pregnancies that delivered fetus structurally normal were considered; 187 women participated in a previous study [19], but six were later excluded due to fetal or maternal pathology. Gestational age was previously determined to the nearest day based on ultrasound biometry.

Blood was collected at arrival from all the participants and immediately centrifugated. During amniocentesis, an aliquot of up to 3-4 mL of AF surplus to clinical requirement was drawn and both plasma and AF samples were stored at $-80{ }^{\circ} \mathrm{C}$ until assay. Measurements of plasma testosterone were available for 181 women and AF testosterone for 167 fetuses. Total plasma testosterone was measured in duplicate aliquots at the Department of Chemistry and Biochemistry of the Faculty of Sciences, University of Lisbon, by standard radio-immunoassay (RIA) using Spectria testosterone RIA® coated tubes from Orion Diagnostica (Finland). The intra- and interassay coefficients of variation of plasma testosterone were $4.5 \%$ and $5.1 \%$, respectively. The concentration of testosterone in the AF was assayed at King's College, London, using a development of the method by Kulle and co-workers [20]. Oasis HLB 1 cc (30 $\mu \mathrm{g})$ extraction cartridges were used for solid phase extraction of the samples with isopropanol. The reconstituted samples were then analyzed by ultra-pressure liquid chromatography followed by mass spectrometry (LCMS/MS).
The digit lengths were measured for 106 NBs (54 females, 52 males) and their mothers. Amniotic testosterone was available for 100 out of these 106 mother-newborn pairs. Photocopies were taken to the ventral surface of the left and right hands of both mother and infant before they left the hospital. With the mother's or nurse's assistance, the measurers placed, one at a time, the palmar surface of both infant's hands on the photocopier plate without pressing hard and provided assistance to their mothers in doing the same. Second copies were made if the landmarks for measuring were not clear. The index, middle, and ring fingers were to be held in an extended position, whereas the thumb and little finger were allowed to be held in a relaxed position. A white cloth cover was used to block light from out of the plate. The finger lengths for the index and ring fingers, respectively 2nd and 4th fingers, were determined, by measuring the distance from the middle of the basal flexion crease proximal to the palm to the tip of the finger, using a vernier caliper measuring to $0.01 \mathrm{~mm}$. On average, the hands were photocopied $38.9 \mathrm{~h}$ (st dev 60.2, median $27.2 \mathrm{~h}$ ) after birth.

All fingers were measured by two measurers who were unaware of each other's results. Repeatability was evaluated by repeated measures ANOVA, where the F ratio evaluates the between-subjects mean squares divided by the error mean squares (i.e. the measurement error in the same hand). The intra-class correlation coefficient $\left(r_{1}\right)$ and the Cronbach's $\alpha$ were also computed. Sample means were compared by independent-samples or paired-samples Student's t tests, as appropriate, multiple regression analysis was used to check association between multiple variables, and a p-value $<0.05$ was routinely considered significant. Numerical data were first checked for normality by examination of their histogram against the empirically expected Normal distribution and by inspection of P-P plots. Amniotic fluid testosterone strayed from normality but logarithmization increased its skewness. In practice, however, our main results are identical with transformed and untransformed data. Statistical analysis was conducted using SPSS version 19.0 (SPSS Inc., Chicago, IL, USA).

\section{Results}

The 106 mothers were on average 37.7 years old (median was 38 , range: 26-43) with mean gestational age at time of sampling of 17.2 weeks (median was 17, range: 15.7-23.6). Average gestation age at birth was 39.4 weeks, with no significant differences between sexes; the NBs were $49 \%$ boys and $51 \%$ girls ( 52 and 54 , respectively). The NB males had an average weight significantly higher ( $t$-test, $\mathrm{p}=$ 0.002 ) than females, respectively, 3399 and $3086 \mathrm{~g}$ (Table 1).

\subsection{Digit ratio}

Repeated measures ANOVA of the 2D:4D ratio in NB hands showed that the ratio of 2D:4D differences between subjects to measurement error was highly significant in both hands (right hand: $\mathrm{F}=$ 95.6, $\mathrm{p}<0.001$; left hand: $\mathrm{F}=93.4, \mathrm{p}<0.001)$. The corresponding intra-class correlations when comparing measurements made by the two observers were $r_{1}=0.89$ and 0.90 (both $p<0.001$ ), respectively, for the right and left hands. The Cronbach's $\alpha$ were 0.94 and 0.95 , respectively, for the right and left hands. We took this as evidence that measurements of the 2D:4D were very similar between observers and there were no directional biases. Henceforth we have used the mean of the 2D:4D ratios available for each hand from the two observers.

The point-biserial correlation between sex and 2D:4D is $r=0.10$ $(p=0.31)$ for the right hand and $r=0.28(p=0.004)$ for the left, with a large overlap between male and female distributions of 2D:4D (Fig. 1). Male NBs had lower mean 2D:4D ratios than females (Table 2) and the differences were significant between left hands (males: 0.927; females: 0.950; $\mathrm{t}=2.95, \mathrm{p}=0.004$; Cohen's $d=0.57$ ) 
Table 1

Descriptive statistics of women undergoing amniocentesis (age and testosterone in plasma), of fetus (gestation age and testosterone in amniotic fluid, AF), and of NBs (age and weight at birth). The last line is time elapsed from delivery till time of hand measurement.

\begin{tabular}{lcccc}
\hline & Mean & St dev & Min & Max \\
\hline Mothers & & & & \\
Age (years) & 37.7 & 2.8 & 26.8 & 43.5 \\
Plasma testosterone - with males (nmol/L) & 1.58 & 0.67 & 0.52 & 3.59 \\
Plasma testosterone - with females (nmol/L) & 1.81 & 0.90 & 0.31 & 4.31 \\
& & & & \\
Fetus and newborn & & & & \\
Gestation age at amniocentesis (weeks) & 17.2 & 1.1 & 15.7 & 23.6 \\
Gestation at birth (weeks) & 39.4 & 1.27 & 34.9 & 41.7 \\
AF testosterone - males (nmol/L) & 0.86 & 0.40 & 0.00 & 1.71 \\
AF testosterone - females (nmol/L) & 0.34 & 0.33 & 0.00 & 1.43 \\
Birth weight - males (g) & 3399 & 507.2 & 2180 & 4610 \\
Birth weight - females (g) & 3086 & 495 & 1725 & 4270 \\
Time till hand photocopy (h) & 38.9 & 60.2 & 2.35 & 541.3 \\
\hline
\end{tabular}

but not between right hands (males: 0.945 ; females: $0.954 ; \mathrm{t}=1.03$, $\mathrm{p}=0.305$; Cohen's $d=0.21)$.

The mean 2D:4D ratios were also compared among mothers. Those who had sons, had lower ratios than those with daughters (Table 2). Once again, the differences were significant between left hands (NB son: 0.959; NB daughter: 0.977; $t=2.77, \mathrm{p}=0.007$ ) but not right hands (NB son: 0.977 ; NB daughter: $0.986 ; \mathrm{t}=1.49, \mathrm{p}=$ 0.14 ), a pattern similar to the one found in NBs. The 2D:4D mean ratios of NB males and females were lower than those of their mothers (Table 2) and the differences were always significant
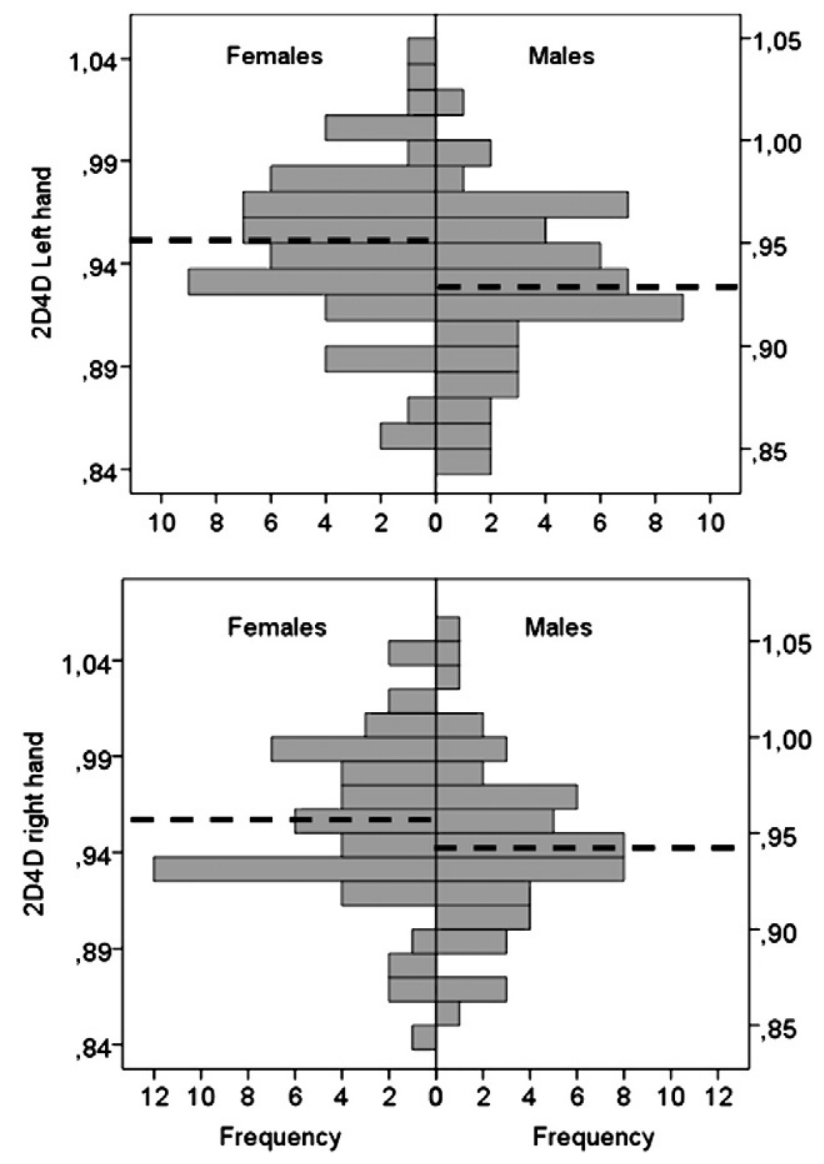

Fig. 1. Histograms of the distributions of the $2 \mathrm{D}: 4 \mathrm{D}$ ratio in the left (top) and right (bottom) hands of 54 female and 52 male NBs. Dashed lines position the mean 2D:4D of each sex.
Table 2

Mean (standard deviation) 2D:4D ratios in newborns and their mothers

\begin{tabular}{llll}
\hline \multirow{2}{*}{ Newborns } & \multicolumn{2}{l}{ 2D:4D mean (st dev) } \\
\cline { 3 - 4 } & & Right hand & Left hand \\
\hline \multirow{3}{*}{ Mothers } & Male $(\mathrm{n}=52)$ & $0.945(0.043)$ & $0.927(0.039)$ \\
& Female $(\mathrm{n}=54)$ & $0.954(0.042)$ & $0.950(0.042)$ \\
& Total $(\mathrm{n}=106)$ & $0.982(0.031)$ & $0.968(0.034)$ \\
& With NB son & $0.977(0.032)$ & $0.959(0.035)$ \\
& With NB daughter & $0.986(0.030)$ & $0.977(0.032)$ \\
\hline
\end{tabular}

$(\mathrm{p}<0.001)$ when evaluated by hand using a paired-sample t-test. For instance, the mean 2D:4D difference between mother and son in the right hand is 0.032 (st dev 0.048) and this difference is significant (paired t-test $\mathrm{t}=4.83, \mathrm{p}<0.001$ ).

We have further looked into the association between the NB 2D:4D and sex, controlling for the influence of the mothers' ratios, using multiple regression with NB 2D:4D as dependent variable and three independent variables: mother's 2D:4D (left and right hands) and sex. Altogether, the two mother's hands account for a significant $\mathrm{R}^{2}=10 \%(\mathrm{p}=0.006)$ of the variability in the NB 2D:4D right hand and addition of sex to the model does not add up a significant contribution, with $\mathrm{R}^{2}$ increasing to only $10.1 \%(\mathrm{p}=0.78)$, confirming the absence of association between sex and the NB right hand. As for the NB left hand, the mother's ratios account for $9.2 \%(p=0.008)$ of 2D:4D variability and the addition of sex to the model is significant, with $\mathrm{R}^{2}$ increasing to $17 \%(\mathrm{p}=0.003)$. The NB left hand thus remains associated with sex when controlling for the mothers' ratios.

The 2D:4D of the mother's right hand was positively correlated with the 2D:4D of both hands of NB females (right hand $r=0.35$, $\mathrm{p}=0.01$; left hand $\mathrm{r}=0.30, \mathrm{p}=0.03$ ) but correlations with males' 2D:4D, although positive, were not significant (Table 3). The 2D:4D of the mother's left hand correlated significantly only with the right hand of NB females $(r=0.28, p=0.04)$. The $2 \mathrm{D}: 4 \mathrm{D}$ were significantly correlated between the right and left hands in NB females $(r=0.54$, $\mathrm{p}<0.001)$ and in the mothers $(\mathrm{r}=0.6, \mathrm{p}<0.001)$ but not in NB males $(\mathrm{r}=0.14, \mathrm{p}=0.32)$.

On average, NB males had longer fingers than females (Table 4). For example, in the right hand, the mean 2D was 25.38 and $24.71 \mathrm{~mm}$, respectively, in males and females. It is possible that this is just because NB males tend to be larger than females, judging from their mean birth weight (Table 1 ), and because birth weight correlates with finger length. We have used regression analysis to determine the mean length of individual fingers to be expected in males if their average birth weight was equal to females. After this correction, however, the expected 2D and 4D mean lengths of males remained longer than those of females (Table 4).

\subsection{Testosterone}

The median of testosterone in the AF was $36 \%$ of the median testosterone in maternal plasma (quartiles: $14-61 \%, n=167$ ) and the two testosterones are not significantly correlated (males: $r=-0.01$, $\mathrm{n}=81, \mathrm{p}=0.93$; females: $\mathrm{r}=0.18, \mathrm{n}=86, \mathrm{p}=0.10$ ). Testosterone in the AF of males was significantly higher (t-test, $\mathrm{p}<0.001, \mathrm{n}=167$ ) than in females, with an average of $0.83 \mathrm{nmol} / \mathrm{L}$ ( $\mathrm{sd} \mathrm{0.40)}$ in the

Table 3

Pearson's correlation coefficient between NBs and their mothers in regard to 2D:4D ratios, by hand and sex. Significant correlations $(p<0.05)$ are signaled with asterisks.

\begin{tabular}{|c|c|c|c|c|c|c|c|c|c|}
\hline & & \multicolumn{4}{|c|}{ NB males } & \multicolumn{4}{|c|}{ NB females } \\
\hline & & Right & $\mathrm{p}$ & Left & $\mathrm{p}$ & Right & $\mathrm{p}$ & Left & $\mathrm{p}$ \\
\hline \multirow[t]{2}{*}{ Mother } & Right & 0.227 & 0.11 & 0.206 & 0.14 & $0.35^{*}$ & 0.01 & $0.30^{*}$ & 0.03 \\
\hline & Left & 0.223 & 0.11 & -0.03 & 0.86 & $0.28^{*}$ & 0.04 & 0.06 & 0.65 \\
\hline
\end{tabular}


Table 4

Observed mean lengths ( $\mathrm{mm}$ ) of the 2D and 4D fingers, by hand and sex. In males are also shown the expected lengths in case the mean weight of males at birth was equal to that of females.

\begin{tabular}{|c|c|c|c|c|c|c|c|}
\hline & \multicolumn{3}{|c|}{ Right hand } & & \multicolumn{3}{|c|}{ Left hand } \\
\hline & Female & $\begin{array}{l}\text { Male } \\
\text { (observ) }\end{array}$ & $\begin{array}{l}\text { Male } \\
\text { (expected) }\end{array}$ & & Female & $\begin{array}{l}\text { Male } \\
\text { (observ) }\end{array}$ & $\begin{array}{l}\text { Male } \\
\text { (expected) }\end{array}$ \\
\hline $2 \mathrm{D}$ & 24.71 & 25.38 & 25.02 & $2 \mathrm{D}$ & 24.30 & 24.89 & 24.53 \\
\hline $4 \mathrm{D}$ & 25.94 & 26.90 & 26.34 & $4 \mathrm{D}$ & 25.62 & 26.87 & 26.49 \\
\hline
\end{tabular}

former and $0.34 \mathrm{nmol} / \mathrm{L}$ (sd 0.31) in the latter (Fig. 2). AF testosterone is negatively correlated with the $2 \mathrm{D}: 4 \mathrm{D}$ ratio in both hands of NB females (Table 5, Fig. 3), but no association is found with 2D:4D in NB males. We shall return to this below, in a more comprehensive account of variability in the NB 2D:4D ratio. AF testosterone does not correlate with gestational age in either sex (males: $r=0.16, n=81$, $\mathrm{p}=0.17$; females: $\mathrm{r}=-0.04, \mathrm{n}=86, \mathrm{p}=0.75$ ).

As for testosterone in maternal plasma, there was no significant difference ( $t$-test, $\mathrm{p}=0.22$ ) between women with male and female fetuses (Fig. 2). In the larger plasma sample $(n=181)$, the mean was $1.60 \mathrm{nmol} / \mathrm{L}(\mathrm{sd} 0.72 \mathrm{nmol} / \mathrm{L}$ ) and $1.80 \mathrm{nmol} / \mathrm{L}(\mathrm{sd} 1.41 \mathrm{nmol} / \mathrm{L}$ ) for males and females, respectively (Table 1). Plasma testosterone exhibits a negative correlation with maternal age in both sexes (male fetus: $r=-0.21, n=90, p=0.05$; female fetus: $r=-0.38, n=97, p<0.001$ ).

Testosterone in the mothers' plasma, exhibits a negative correlation with all four 2D:4D ratios in NBs (Table 5), but none is statistically significant. When testosterone in the mothers' plasma increases, the 2D:4D of the NB tends to decrease, but our sample size split by sexes may have been insufficient to capture statistical significance. Only when sexes are pooled does plasma testosterone become significantly correlated with $2 \mathrm{D}: 4 \mathrm{D}$ in the right hand $(\mathrm{r}=-0.2, \mathrm{p}=0.04$, $\mathrm{n}=106$ ). Plasma testosterone is also negatively correlated with the mother's 2D:4D ratio in both hands (right: $r=-0.36, p=0.011$; left: $r=-0.37, p=0.008$ ) when their fetus is male, but not when the fetus is female (right: $r=0.16, p=0.26$; left: $r=0.06, p=0.65$ ).

We have next examined the proportion of variability in the NB ratios (by sex and hand) accounted by the mother's ratios and by testosterone, using a hierarchical regression model where the NB $2 \mathrm{D}: 4 \mathrm{D}$ is the dependent variable and independent variables are the mother's 2D:4D ratios (two variables: left, right), AF testosterone, and maternal testosterone.

The models with the mothers' 2D:4D and AF testosterone account for a significant proportion of the variability in the $2 \mathrm{D}: 4 \mathrm{D}$ ratio of $\mathrm{NB}$ females $\left(R^{2}=24.9 \%\right.$ and $25.3 \%$, respectively right and left hands). Table 6 indicates that the mother's ratios, by themselves, are quite
Table 5

Pearson's correlation coefficients ( $\mathrm{r}$ ) between testosterone $(\mathrm{T})$ in the amniotic fluid and in the mother's plasma and 2D:4D ratios in the newborns, by hand and sex. Asterisk signals significant correlation.

\begin{tabular}{lcccccc}
\hline & \multicolumn{3}{c}{ Right hand } & & \multicolumn{2}{c}{ Left hand } \\
\cline { 3 - 4 } \cline { 6 - 7 } & & Males & Females & & Males & Females \\
\hline T in AF & $\mathrm{r}$ & 0.14 & -0.24 & & 0.004 & -0.3 \\
& $\mathrm{n}$ & 49 & 51 & & 49 & 51 \\
$\mathrm{~T}$ T in plasma & $\mathrm{p}$ & 0.35 & 0.09 & & 0.98 & $0.03^{*}$ \\
& $\mathrm{r}$ & -0.2 & -0.24 & & -0.11 & -0.1 \\
& $\mathrm{n}$ & 52 & 54 & & 52 & 54 \\
& $\mathrm{p}$ & 0.15 & 0.09 & & 0.44 & 0.49 \\
\hline
\end{tabular}

significant (15.3\% and $13.5 \%$ of the variance, respectively, right and left) but addition of AF testosterone improves the model significantly, with an additional $9.6 \%$ and $11.8 \%$ for the right and left hands, respectively, always with negative regression coefficients. The addition of plasma testosterone does not improve the models significantly. As for NB males, the models do not account for the variability observed in the 2D:4D ratio of either hand. The amount of variance explained by the complete model was $8.4 \%$ and $1.2 \%$, respectively, for the right and left NB hands (Table 6), and none was significant.

\section{Discussion}

Our data shows that the 2D:4D dimorphism is already present at birth, confirming previous reports that it shows as early as at 14 weeks of gestation [16]. As expected, males had lower mean 2D:4D ratio than females, but like previous authors $[2,14]$ we found that the difference is subtle and there is a large overlap of 2D:4D distributions between sexes (Fig. 1). In our sample of NB infants, sex dimorphism was significant for the left but not for the right hand. This contrasts with studies which sampled individuals older than two years and reported a greater 2D:4D sex difference for the right hand [21-23]. Our results are from individuals in between the preand postnatal testosterone surges, and because we have not find significant dimorphism in the right hand, we suggest that postnatal androgen exposure is rather influential for the accentuation of dimorphism and stabilization of 2D:4D ratio in the right hand. A recent study [17], working only with right hand photocopies of children between 3 and 5 months of age, also did not find significant 2D:4D differences between sexes. Likewise, newborns in the Knickmeyer et al. [18] study had a tendency to show sexual dimorphism in 2D:4D, but differences were not significant. The postnatal testosterone exposure thus appears determinant for the right hand ratio later observed in life.
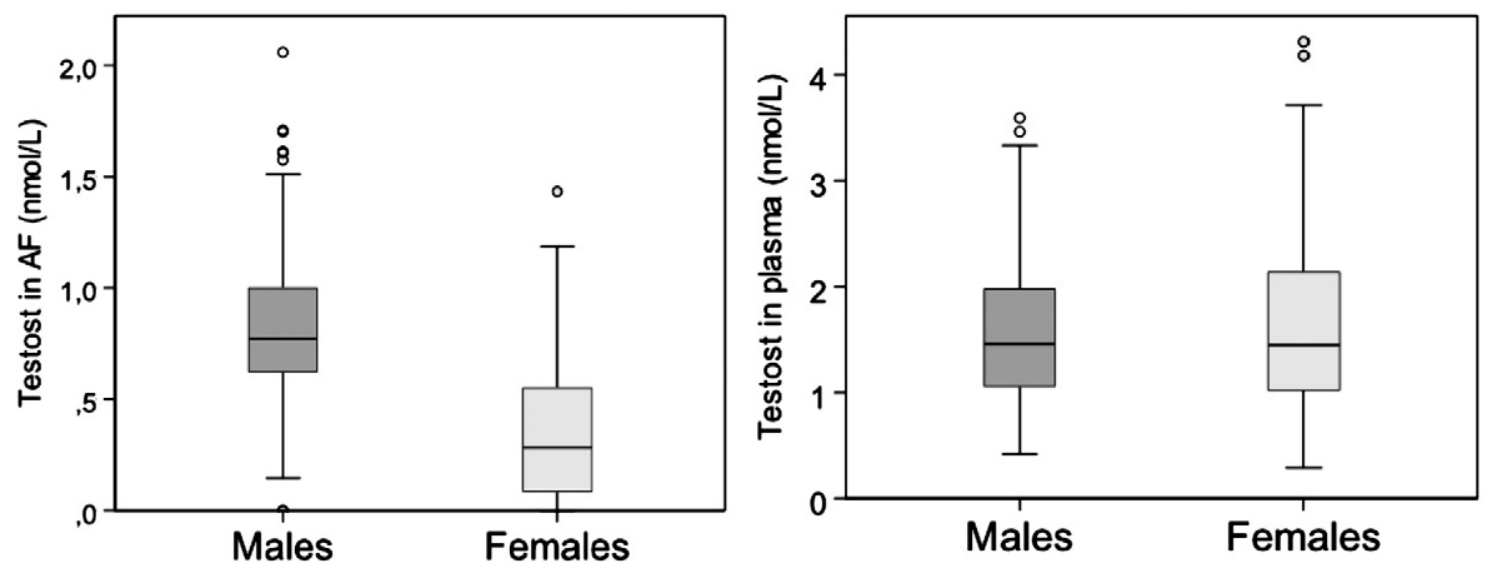

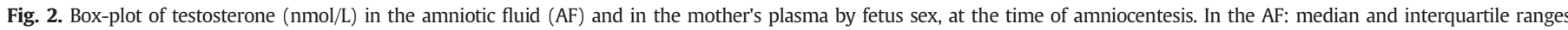

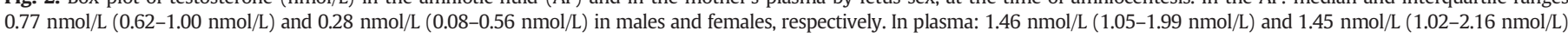
in males and females, respectively. 

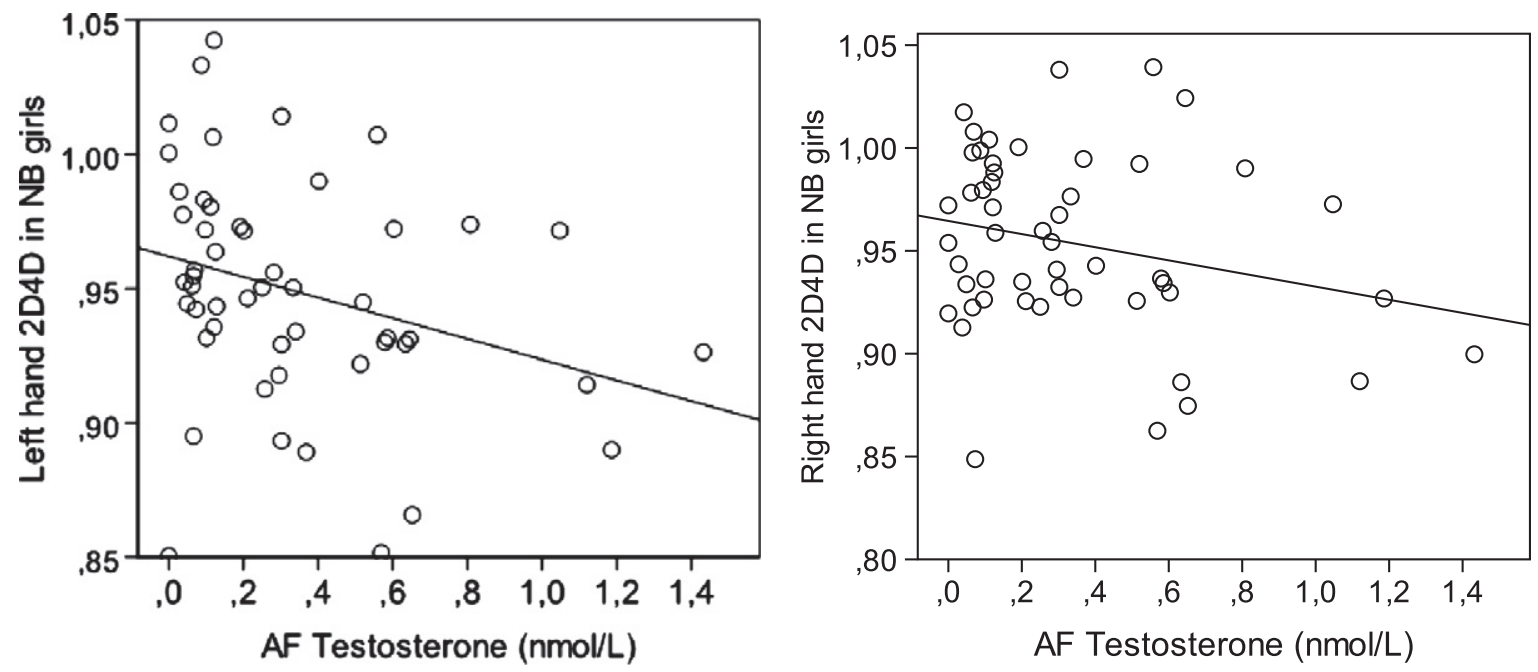

Fig. 3. Negative associations between $A F$ testosterone and the $2 D: 4 D$ ratio in the left $(r=-0.3, p=0.03)$ and the right $(r=-0.24, p=0.09)$ hands of $N B$ females.

Mothers who had sons had lower 2D:4D ratios than those with daughters ( $p=0.007$ and 0.14 , for left and right hands, respectively) (Table 2). This is consistent with a previous report that the 2D:4D of parents correlates negatively with the proportion of their male offspring [24] and with a broader hypothesis stating that the sex of offspring in mammals is associated with hormone concentrations of both parents at time of conception $[25,26]$ with high concentrations of testosterone (and low 2D:4D) increasing the likelihood of having a son.

The mother's 2D:4D was a significant predictor of the newborn 2D:4D ratio in both hands (right: $R^{2}=10 \%, p=0.006$; left: $R^{2}=$ $9.2 \%, \mathrm{p}=0.008$ ) after controlling for sex. AF testosterone was also found to be negatively correlated with the 2D:4D in NB females, but not in males. We have then examined the moderating effect of sex in these associations by dividing the NBs in two groups, according to sex, and regressing the NB 2D:4D ratio on the mother's ratios (left and right hands) and on testosterone in each group (Table 6). We have found that the mother's 2D:4D are significant predictors of 2D:4D in NB daughters, but not in sons. In NB daughters, a significant proportion of regression residuals can still be accounted by AF testosterone, stemming from a negative partial correlation between NB ratios and AF testosterone. The percentages of variability accounted by the mother ratios and AF testosterone were, respectively, $15.3 \%$ and $9.6 \%$ in the right hand, and $13.5 \%$ and $11.8 \%$ in the left hand, suggesting a balanced contribution of genetics and testosterone to the determination of 2D:4D in girls at birth. We have not found similar associations between the 2D:4D ratios of NB males and their mother's 2D:4D or testosterone, as the models did not account for more than $8.4 \%$ and $1.2 \%$ of variability in the right and left hands, respectively (Table 6). The lack of correlation with the mother's 2D:4D could result from a high level of testosterone in the prenatal environment during digit-forming stages of embryonic development. Digit development is a complex process, involving the regulation of genes' expression by testosterone and estrogen [10] and high testosterone might induce a level of individual variability capable of breaking down correlation between males' 2D:4D and their mothers' 2D:4D. We also have not found correlation between males' 2D:4D and $\mathrm{AF}$ testosterone. We hypothesize that because the male fetus is normally subjected to higher levels of testosterone, levels above average do not have any significant impact in further decreasing the 2D:4D ratio. Brown et al. [5] suggested that androgen receptors may saturate in normal male fetus or else stay within specific ranges so that additional levels of androgens would not be as influential for the $2 \mathrm{D}: 4 \mathrm{D}$ as in girls.

We have found that NB males had, in both hands, mean longer fingers than females, corroborating a tendency previously pointed out for 3 to 5 month-olders [17] (Table 4). Our data also support claims that 2D:4D increases from birth to adulthood [14-17], as mothers had significantly larger 2D:4D than their NBs (Table 2) for both sexes and hands. Postnatal developmental processes should thus be involved in the determination of 2D:4D sexual dimorphism later found in adults.

The finding of sexual dimorphism at birth has been interpreted as evidence for prenatal testosterone action and we expected to find higher testosterone levels associated with lower, masculinized, 2D:4D in NBs [2]. Indeed, we have found weak negative correlation of testosterone levels in the mother's plasma and 2D:4D ratios in both sexes and hands, but our sample (52 males, 54 females) apparently lacked power to reach statistical significance.

It is commonly accepted that androgen levels, including testosterone, show a substantial reduction in women that approach the menopause, usually about age 50 , and in the years immediately after

Table 6

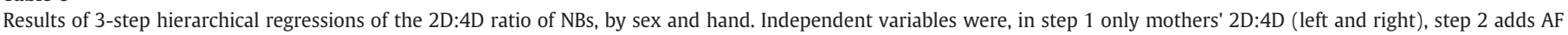

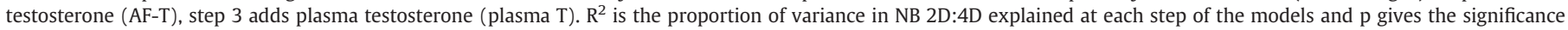
of the increment $\Delta R^{2}$ in variance explained, signaled by ${ }^{*}<0.05$ and ${ }^{*}<0.01$.

\begin{tabular}{|c|c|c|c|c|c|c|c|}
\hline NB females & $\mathrm{R}^{2}$ & $\Delta \mathrm{R}^{2}$ & $\mathrm{p}$ & NB males & $\mathrm{R}^{2}$ & $\Delta \mathrm{R}^{2}$ & $\mathrm{p}$ \\
\hline NB right hand & & & & NB right hand & & & \\
\hline Mother 2D:4D & 0.153 & 0.153 & $0.02 *$ & Mother 2D:4D & 0.063 & 0.063 & 0.23 \\
\hline Mother 2D:4D + AF-T & 0.249 & 0.096 & $0.02^{*}$ & Mother 2D:4D + AF-T & 0.074 & 0.011 & 0.46 \\
\hline Mother 2D:4D + AF-T + plasma T & 0.305 & 0.056 & 0.06 & Mother 2D:4D + AF-T + plasma T & 0.084 & 0.009 & 0.51 \\
\hline NB left hand & & & & NB left hand & & & \\
\hline Mother 2D:4D & 0.135 & 0.135 & $0.03^{*}$ & Mother 2D:4D & 0.011 & 0.011 & 0.77 \\
\hline Mother 2D:4D + AF-T & 0.253 & 0.118 & $0.01^{* *}$ & Mother 2D:4D + AF-T & 0.012 & 0.001 & 0.85 \\
\hline Mother 2D:4D + AF-T + plasma $\mathrm{T}$ & 0.261 & 0.008 & 0.48 & Mother 2D:4D + AF-T + plasma $\mathrm{T}$ & 0.012 & 0.000 & 0.99 \\
\hline
\end{tabular}


$[27,28]$. The pregnant women in our study ranged between 26 and 43 years old but still we have found evidence for a decrease in testosterone irrespective of the fetus' sex. There was however no such association between mother's age and testosterone in the AF.

Like previous authors [29] we did not find significant differences in mean testosterone concentrations in maternal plasma between the two fetus sexes. However, testosterone in the AF was significantly higher in males than in females (Fig. 2). Unlike Sarkar and co-workers [29], we did not find a significant correlation between levels of testosterone in the AF and in the maternal plasma $(r=-0.01, p=0.93 ; r=$ $0.11, p=0.30$, respectively, in males and females). Altogether, these results are suggestive that there is no significant transport of testosterone between the mother and the fetal environment. The main source of testosterone in AF is likely the fetus itself.

\section{Conclusion}

Sexual dimorphism is present at birth, with a large overlap between sexes, but the tendency for being more accentuated in the right hand, as opposed to the left, was not confirmed, suggesting that postnatal testosterone exposure and developmental processes are determinant for the right hand ratio and sexual dimorphism later observed in life. Postnatal development is also likely to explain why the 2D:4D ratios in NBs are significantly smaller than those of their mothers.

Mothers who had sons, had significantly lower 2D:4D ratios than those who had daughters, supporting claims that offspring sex is associated with hormone concentrations in parents around conception. The mother's 2D:4D ratios are significantly correlated with their daughters' 2D:4D ratios, and AF testosterone explains a significant proportion of the remaining variability observed in female NBs. The same was not observed for male NBs though, suggesting that high prenatal testosterone levels in male fetus may lead their 2D:4D ratios to differ from their mothers' due to high individual variability.

\section{Conflict of interest}

The authors declare no conflict of interest.

\section{Acknowledgments}

We acknowledge Arium-Sistemas de Diagnóstico Lda for assuring quality control in all the assays in Lisbon, Miss Olah Hakim (King's College, London), for her help in the laboratory, and Professor Luis Pereira da Silva for support. We also thank two anonymous reviewers for comments that helped to improve the manuscript.

\section{References}

[1] Phelps VR. Relative index finger length as a sex-influenced trait in man. Am J Hum Genet 1952;4:72-89.

[2] Manning JT, Scut D, Wilson J, Lewis-Jones DI. The ratio of 2nd to 4th digit length, a predictor of sperm numbers and concentrations of testosterone, luteinizing hormone and oestrogen. Hum Reprod 1998;13:3000-4.
[3] Manning JT, Trivers RL, Thornhill R, Singh D. The 2nd:4th digit ratio and asymmetry of hand performance in Jamaican children. Laterality 2000;5:121-32.

[4] Robinson SJ, Manning JT. Ratio of 2nd to 4th digit length and male homosexuality. Evol Hum Behav 2000;21(5):333-45.

[5] Brown WM, Hines M, Fane BA, Breedlove SM. Masculinized finger length patterns in human males and females with congenital adrenal hyperplasia. Horm Behav 2002;42:380-6.

[6] Okten A, Kalyoncu M, Yaris N. The ratio of second- and fourth digit lengths and congenital adrenal hyperplasia due to 21-hydroxylase deficiency. Early Hum Dev 2002;70:47-54.

[7] van Anders SM, Vernon PA, Wilbur CJ. Finger-length ratios show evidence of prenatal hormone-transfer between opposite-sex twins. Horm Behav 2006;49:315-9.

[8] Voracek M, Dressler SG. Digit ratio (2D:4D) in twins: heritability estimates and evidence for a masculinized trait expression in women from opposite sex pairs. Psychol Rep 2007:100:115-26.

[9] Manning JT, Bundred PE, Newton DJ, Flanagan BF. The second to fourth digit ratio and variation in the androgen receptor gene. Evol Hum Behav 2003;24:399-405.

[10] Zheng Z, Cohn MJ. Development basis of sexually dimorphic digit ratios. PNAS 2011;108:16289-94.

[11] Garn SM, Burdi AR, Babler WJ, Stinson S. Early prenatal attainment of adult metacarpal-phalangeal rankings and proportions. Am J Phys Anthropol 1975;43: 327-32.

[12] Malas MA, Dogan S, Evcil EH, Desdicioglu K. Fetal development of the hand, digits and digits ratio (2D:4D). Early Hum Dev 2006;82(7):469-75.

[13] Prince FP. The triphasic nature of Leydig cell development in humans, and comments on nomenclature. J Endocrinol 2001;168:213-6.

[14] McIntyre MH, Ellison PT, Lieberman DE, Demerath E, Towne B. The development of sex differences in digital formula from infancy in the Fels Longitudinal Study. Proc R Soc B 2005;272:1473-9.

[15] Trivers R, Manning J, Jacobson A. A longitudinal study of digit ratio (2D:4D) and other finger ratios in Jamaican children. Horm Behav 2006;49:150-6.

[16] Galis F, Ten Broek CMA, Van Dongen S, Wijnaendts LCD. Sexual dimorphism in the prenatal digit ratio (2D:4D). Arch Sex Behav 2010;39:57-62.

[17] McIntyre MH, Alexander GM. Sex differences in the fingers of 3 to 5 month old infants do not predict concurrent salivary testosterone levels. Early Hum Dev 2011;87:349-51.

[18] Knickmeyer RC, Woolson S, Hamer RM, Konneker T, Gilmore JH. 2D:4D ratios in the first 2 years of life: stability and relation to testosterone exposure and sensitivity. Horm Behav 2011;60:256-63.

[19] Ventura T, Gomes MC, Carreira T. Cortisol and anxiety response to a relaxing intervention on pregnant women awaiting amniocentesis. Psychoneuroendocrinology 2012;37:148-56.

[20] Kulle AE, Riepe FG, Melchior D, Hiort O, Holterhus PM. A novel ultrapressure liquid chromatography tandem mass spectrometry method for the simultaneous determination of androstenedione, testosterone, and dihydrotestosterone in pediatric blood samples: age- and sex-specific reference data. J Clin Endocrinol Metab 2010;95(5):2399-409.

[21] Williams TJ, Pepitone ME, Christensen SE, Cooke BM, Huberman AD, Breedlove NJ et al. Finger-length ratios and sexual orientation. Nature 2000;404:455-6.

[22] Manning JT, Churchill AJG, Peters M. The effects of sex, ethnicity, and sexual orientation on self-measured digit ratio (2D:4D). Arch Sex Behav 2007;36:223-33.

[23] Gobrogge KL, Breedlove SM, Klump KL. Genetic and environmental influences on 2D:4D finger length ratios: a study of monozygotic and dizygotic male and female twins. Arch Sex Behav 2008;37:112-8.

[24] Manning JT, Martin S, Trivers RL, Soler M. 2nd to 4th digit ratio and offspring sex ratio. J Theor Biol 2002;217:93-6.

[25] James WH. Evidence that mammalian sex ratios at birth are partially controlled by parental hormone levels at the time of conception. J Theor Biol 1996;180:271-86.

[26] James WH. Further evidence that mammalian sex ratios at birth are partially controlled by parental hormone levels around the time of conception. Hum Reprod 2004;19:1250-6.

[27] Meston CM. Aging and sexuality. In: successful agingWest J Med 1997;167: 285-90.

[28] Reckelhoff JF, Yanes LL, Iliescu R, Fortepiani LA, Granger JP. Testosterone supplementation in aging men and women: possible impact on cardiovascular-renal disease. Am J Physiol Renal Physiol 2005;289:F941-8.

[29] Sarkar P, Bergman K, Fisk NM, O'Connor TK, Glover V. Amniotic fluid testosterone: relationship with cortisol and gestational age. Clin Endocrinol 2007:67:743-7. 